\title{
Association of Renal and Bladder Ultrasonography Findings with Urinary Tract Infection Recurrence, High- Grade Vesicoureteral Reflux, and Renal Scarring
}

\author{
Hye Won Park, M.D., \\ Hyeil Jin, M.D., \\ Su Jin Jeong, M.D., \\ Jun Ho Lee, M.D. \\ Department of Pediatrics, $\mathrm{CHA}$ \\ Bundang Medical Center, $\mathrm{CHA}$ \\ University, Seongnam, South Korea
}

Corresponding author: Jun Ho Lee, M.D. Department of Pediatrics CHA Bundang Medical Center, CHA University 351

Yatap-dong, Bundang-gu, Seongnam-si, Gyeonggi-do 463-712, Korea

Tel: +82-10-3180-6511

Fax: +82-31-780-5011

E-mail:naesusana@gmail.com

Co-corresponding author: Su Jin Jeong, M.D. Department of Pediatrics CHA Bundang Medical Center, CHA University 351

Yatap-dong, Bundang-gu, Seongnam-si, Gyeonggi-do 463-712, Korea

Tel: +82-10-9132-8839

Fax: +82-31-780-3452

E-mail:jinped@cha.ac.kr

Received: 16 June 2015

Revised: 7 July 2015

Accepted: 15 July 2015
Introduction: This study investigated whether renal and bladder ultrasonography (RBUS) findings performed in children with the first incidence of febrile urinary tract infection (UTI) can predict UTI recurrence, high-grade vesicoureteral reflux (high-grade VUR), or acquired renal scarring (aRS).

Methods: In all, 917 children who were admitted to our hospital from January 2001 to October 2010, owing to the first incidence of febrile UTI were enrolled in this study. All children underwent RBUS during admission. The mean followup was 7.9 months (standard deviation $[S D] \pm 13.3$ ). UTI recurrence rates were calculated according to various clinical parameters. By using bivariate and multiple logistic regression analyses, we determined whether age, sex, abnormal RBUS findings, abnormal dimercaptosuccinic acid renal scan findings, or RBUS findings parameters were predictive of UTI recurrence, high-grade VUR, or aRS.

Results: On RBUS, hydronephrosis and congenital anomaly of the kidney and urinary tract significantly predicted UTI recurrence. A small kidney, hydroureter, hydronephrosis, cortical thinning, and increased parenchymal echogenicity significantly predicted high-grade VUR. However, their odds ratios (OR) are low compared to normal RBUS findings (recurrent UTI: OR 0.432 and 0.354 vs. 0.934 , respectively, high-grade VUR: $.019,0.329,0.126,0.058$, and 0.188 vs. 2.082, respectively). No RBUS findings significantly predicted aRS. Recurrent UTI, highgrade VUR, and abnormal RBUS findings significantly predicted aRS (OR of 4.80, 4.61 , and 2.58 , respectively).

Conclusion: RBUS is necessary to exclude severe congenital renal scarring, obstructive uropathy, and renal abscess at the first incidence of febrile UTI and is helpful in determining the need for subsequent clinical imaging.

Key words: Renal sonogram, Urinary tract infection recurrence, High-grade vesicoureteral reflux, Acquired renal scarring

\section{Introduction}

The purpose of renal and bladder ultrasonography (RBUS) in the first episode of febrile urinary tract infection (UTI) is to detect anatomic abnormalities that require further evaluation, e.g., additional imaging or urologic consultation ${ }^{1)}$. RBUS also evaluates the renal parenchyma and renal size that can be used to monitor renal growth ${ }^{1)}$. The timing of RBUS depends on the 
clinical scenario. RBUS performed early in the course of an acute infection can be misleading; animal studies have demonstrated that dilation due to Escherichia coli endotoxin during acute infection can be confused with hydronephrosis, pyonephrosis, or obstruction ${ }^{2}$. Changes in kidney size and shape and increased echogenicity of the renal parenchyma attributable to edema also are common during acute infection.

The American Association of Pediatrics recommended in 2011 that voiding cystourethrography (VCUG) should not be performed routinely after the first incidence of febrile UTI; VCUG is indicated if RBUS reveals hydronephrosis, scarring, or other findings that would suggest either highgrade vesicoureteral reflux (VUR) or obstructive uropathy as well as in other atypical or complex clinical circumstances (evidence quality B) ${ }^{1)}$.

Most infants with febrile UTI have been admitted to secondary or tertiary hospitals because of concerns about acquired renal scarring or urosepsis and underwent RBUS during acute infection. Mild to moderate hydronephrosis is often detected on RBUS in children with UTIs, especially in young infants. Severe congenital scarring or obstructive uropathy is uncommon; detection of these findings is an indication for a second imaging study. No definitive literature exists regarding whether RBUS findings are associated with UTI recurrence, presence of high-grade VUR, or acquired renal scarring.

This study aimed to investigate whether RBUS findings in children experiencing the first incidence of febrile UTI can predict UTI recurrence, high-grade VUR, or acquired renal scarring, and whether RBUS findings can indicate whether subsequent imaging studies are needed.

\section{Materials and Methods}

This study included 1003 children who were admitted to our hospital from January 2001 to October 2010, owing to their first incidence of febrile UTI. In all, 86 children in this group who did not undergo RBUS were excluded; 917 children were enrolled in this study. Febrile UTI at admission was defined as follows: fever $\geq 38^{\circ} \mathrm{C}$, abnormal urinalysis, positive C-reactive protein (CRP) results $(>0.3$ $\mathrm{mg} / \mathrm{dL}$ ), significant bacteriuria, and absence of any other fever etiology. Abnormal urinalysis was defined as pyuria [ $>5$ white blood cells/high-power field (HPF)]; positive leukocyte esterase; and one or more positive findings of protein, nitrite, or red blood cells. Significant bacteriuria was defined as the presence of $>1 \times 10^{5}$ colony-forming units/HPF in a single-strain isolate. Urine samples during midstream urine were collected in children over 2 and by using the clean catch bag method in young children under 2. All enrolled children underwent RBUS within 48 hours after admission. Dimercaptosuccinic acid (DMSA) renal scan, and VCUG are recommended upon admission. A follow-up DMSA scan was performed in children with positive DMSA results at least 6 months after admission.

Abnormal US findings were defined as hydronephrosis [HN; grade 1: the renal pelvis only is visualized, grade 2: a few but not all calices are identified in addition to the renal pelvis, grade 3: all calices are seen, grade 4: the involved kidney had parenchymal thinning, as defined by the Society for Fetal Urology (SFU) $]^{3)}$, hydroureter, small or hypoplastic kidney (defined as a kidney length $\geq 2$ standard deviations below average for patient age $)^{4}$, swelling or thinning of renal parenchyma, increased renal parenchymal echogenicity, poor differentiation of corticomedullary junction, renal pelvic wall thickening, bladder wall thickening, or other congenital anomaly of the kidney and urinary tract (CAKUT). Positive DMSA findings were defined as small kidney, focally or diffusely decreased cortical uptake, or loss of renal contour. Congenital renal scarring defined by small kidney with decreased relative renal uptake of less than $40 \%$ with a smooth renal contour without focal uptake defects on DMSA scan. Acquired renal scarring defined as presence of any focal uptake defect on follow-up DMSA scan. High-grade VUR in VCUG were defined by grade 4 and 5 VUR. A single pediatric radiologist assessed the RBUS scans; the DMSA scans were interpreted by both a nuclear medicine consultant and a pediatric nephrologist.

Duration of follow-up was determined according to the duration of outpatient clinic follow-up. Person-years were calculated in children with an episode of UTI recurrence by subtracting age at study start date from age at the last episode of recurrent UTI; they were calculated in children without an episode of UTI recurrence by subtracting age at study start date from age at study end date. UTI recurr- 
ences were confirmed with retrospective chart reviews as well as direct contacts by telephone with patients' parents. All data were analyzed retrospectively.

\section{Statistical analysis}

All variables are presented as the mean \pm standard deviation (SD), and continuous variables were analyzed using the Mann-Whitney U test (2-tailed probability). Correlations were analyzed using the Pearson correlation coefficient, Spearman's rank correlation coefficient, and Kendall tau correlation coefficient (two-tailed probability). To determine whether RBUS findings predict UTI recurrence, highgrade VUR, or acquired renal scarring in children with the first febrile incidence UTI, bivariate and multiple logistic regression analyses (MLRA) were used. Statistical analysis was performed using SPSS statistics 20 (SPSS Inc., Chicago, IL, USA). Statistical significance was defined as $P<0.05$.

\section{Results}

Demographic findings of the study population are shown in Table 1. UTI recurrence rate in this study was $12.9 \%$. VUR was detected in $20.6 \%$ and high-grade VUR was detected in $50.7 \%$ among VUR patients, respectively. Congenital renal scarring was detected in 16 patients (2.0\%). Acquired renal scarring was detected in $50 \%$ of 132 children who underwent a follow-up DMSA scan. The mean followup period was $7.9 \pm 13.3$ months. Duplex kidney, rotational anomaly of the kidney, renal cyst, polycystic kidney, multicystic dysplastic kidney, urachal remnant, renal abscess, and nephrocalcinosis were detected in $15,1,1,3,2,1,3$, and 1 patients, respectively.

The mean age of male patients was less than that of female patients ( 7.3 vs. 17.1 months, $P<0.001$ ). The recurrence rate of UTI was not meaningfully different between males and females $(P>0.05)$. In this study population, $2.2 \%$ children experienced 3 or more episodes of recurrent UTI. The recurrence rate of UTI was high in children with operation of VUR, high-grade VUR, grade III VUR, VUR, congenital renal scarring, less than $40 \%$ of relative renal uptake on DMSA scan, abnormal DMSA scan findings, and abnormal RBUS findings in sequence $(62.5 \%, 59.7 \%$, $46.7 \%, 41.5 \%, 40.9 \%, 35.5 \%, 20 \%$, and $18.7 \%$, respectively)
(Table 1).

According to RBUS findings, MLRA of predicting UTI recurrence, high-grade VUR, and acquired renal scarring is shown in Table 2. By MLRA, hydronephrosis and CAKUT significantly predicted UTI recurrence in the future $(\mathrm{P}=0.034$ and 0.048 , respectively). By correlation analysis, hydroureter, hydronephrosis, and CAKUT were correlated with UTI recurrence ( $\mathrm{P}=0.045,0.000$, and 0.012 , respectively). By MLRA, presence of a small kidney, hydroureter, hydronephrosis, increased parenchymal echogenicity, and cortical thinning were significant risk factors associated with high-grade VUR ( $\mathrm{P}=0.000,0.002,0.000,0.005$, and 0.012 , respectively). By correlation analysis, presence of a small kidney, hydroureter, hydronephrosis, and cortical thinning were correlated with the presence of high-grade $\operatorname{VUR}(\mathrm{P}=0.000,0.003,0.000$, and 0.000). By MLRA, none of RBUS findings predicted acquired renal scarring. By correlation analysis, CAKUT only was correlated with the presence of acquired renal scarring $(\mathrm{P}=0.006)$.

Table 3 showed bivariate logistic regression analysis with odds ratio (OR) to predict UTI recurrence, highgrade VUR, and acquired renal scarring according to various parameters, compared with abnormal renal sonogram findings. The presence of VUR and high-grade VUR was significant risk factors for UTI recurrence $(\mathrm{P}=0.045$ and $\mathrm{P}=0.003$, respectively). Abnormal RBUS findings, abnormal DMSA scan findings, and UTI recurrence significantly predicted high-grade VUR ( $\mathrm{P}=0.024,0.003$, and 0.001; ORs of 2.64, 5.53, and 4.27, respectively). Abnormal RBUS findings, high-grade VUR, and UTI recurrence were significant factors for prediction of acquired renal scarring $(P=0.031,0.017$, and 0.002 ; ORs of $2.58,4.64$, and 4.82 , respectively).

\section{Discussion}

Recurrent UTI and high grade VUR are widely known risk factors of acquired renal scarring in children ${ }^{5,6}$. Generally, the recurrence rate of UTI is about $10-20 \%{ }^{7}$. Therefore, at least $80 \%$ of children who experience the first incidence of febrile UTI are unlikely to experience recurrent UTI, further imaging studies, and suspected progressive chronic renal damage. The exceptions include 
Table 1. Baseline clinical characteristics of study population

\begin{tabular}{|c|c|c|c|c|}
\hline & & Number of patients & RR (\%) & $\mathrm{RE}_{\mathrm{UT}} / \mathrm{PY}$ \\
\hline \multirow{3}{*}{ Gender } & Total & 917 & 12.9 & 0.034 \\
\hline & Male & 590 & 12.9 & 0.034 \\
\hline & Female & 327 & 12.8 & 0.031 \\
\hline \multirow{5}{*}{$\begin{array}{l}\text { Age of Diagnosis } \\
\text { (months) }\end{array}$} & Mean & $10.8( \pm 18.6)$ & & \\
\hline & Male & $7.3( \pm 11.5)$ & & \\
\hline & Female & $17.1( \pm 25.8)$ & & \\
\hline & $\leq 2$ year-old & 831 & 11.0 & \\
\hline & $>2$ year-old & 86 & 19.8 & \\
\hline \multirow{3}{*}{ Number of UTI episodes } & Total & 118 & & \\
\hline & 2 & 71 & & \\
\hline & 3 or more & 20 & & \\
\hline \multirow{3}{*}{ RBUS results } & Total & 917 & & \\
\hline & Normal & 527 & 6.6 & 0.025 \\
\hline & Abnormal & 390 & 18.7 & 0.048 \\
\hline \multirow{4}{*}{ DMSA scan results } & Total & 797 & & \\
\hline & Normal & 427 & 9.8 & 0.032 \\
\hline & Abnormal & 370 & 20.0 & 0.059 \\
\hline & Cortical uptake $<40 \%$ & 31 & 35.5 & 0.090 \\
\hline \multirow{7}{*}{ VCUG results } & Total & 690 & & \\
\hline & No VUR & 548 & 8.4 & 0.025 \\
\hline & VUR & 142 & 41.5 & 0.130 \\
\hline & Grade I-II & 47 & 5.0 & 0.024 \\
\hline & Grade III & 30 & 46.7 & 0.102 \\
\hline & Grade IV-V & 72 & 59.7 & 0.254 \\
\hline & Operation of VUR & 24 & 62.5 & 0.545 \\
\hline
\end{tabular}

DMSA, dimercaptosuccinic acid; RBUS, renal sonogram findings; REUTI/PY, recurrent episodes of UTI per person-year; RR, recurrence rate of UTI; UTI, urinary tract infection; VCUG, voiding cystourethrogram; VUR, vesicoureteral reflux;

cases accompanied by severe congenital renal scarring even though there was a report ${ }^{6)}$ that earlier detection of high-grade VUR was more associated with chronic renal injury in the future. Fortunately, severe congenital renal scarring could be leached out by RBUS in the form of small or dysplastic kidney.

It is unclear whether early detection of acquired renal scarring or high-grade VUR is more important. However, the authors suggest that early detection of the presence of acquired renal scarring or recurrent UTI are more important because these are more sensitive indicators for surgical management of VUR in children than early detection of high-grade VUR itself, since there is no current prophylactic approach for recurrent UTI in children with highgrade VUR.

Soliman et al. reported that increased resistivity index, pulsatility index, and decreased diastolic/systolic ratio in both arcuate and interlobar arteries on renal duplex Doppler ultrasonography were observed in recurrent UTI patients and scarred renal units ${ }^{8}$. However, renal Doppler sonogram is not a practical method in uncooperative young children. Katzir et al. reported that neonates with extra renal pelvis had an increased UTI incidence unrelated to VUR within the first two years ${ }^{9}$. In this study, extra renal pelvis was included as a parameter of hydronephrosis. Juliano et al. reported that $15 \%$ of children experiencing UTI with normal RBUS results had recurrent UTIs and that $7 \%$ of them experienced surgical intervention ${ }^{10)}$. In this study, $6.6 \%$ of UTI children with normal RBUS results had recurrent UTI. Among abnormal RBUS findings, hydronephrosis and CAKUT were significant in prediction of recurrent UTI. However, their ORs (0.432 and 0.354 ) were low compared to cases with normal RBUS findings (OR 0.934). Therefore, children with hydronephrosis or CAKUT at 
Table 2. Multiple logistic regression analysis of prediction of urinary tract infection recurrence, high-grade vesicoureteral reflux, and acquired renal scarring based on renal sonogram findings.

\begin{tabular}{|c|c|c|c|c|c|c|c|c|c|c|}
\hline \multirow[t]{2}{*}{ RBUS } & \multirow[t]{2}{*}{ NO. } & \multirow[t]{2}{*}{ rUTI } & \multicolumn{2}{|c|}{ rUTI } & \multirow[t]{2}{*}{ HGVUR } & \multicolumn{2}{|c|}{ HGVUR } & \multirow[t]{2}{*}{ aRS } & \multicolumn{2}{|c|}{ aRS } \\
\hline & & & OR & $95 \% \mathrm{Cl}$ & & OR & $95 \% \mathrm{Cl}$ & & OR & $95 \% \mathrm{Cl}$ \\
\hline Small or hypoplastic & 7 & 0.573 & 0.516 & $0.052-5.138$ & $0.000^{*}$ & 0.019 & $0.002-0.149$ & - & $2.546 \mathrm{E}-9$ & $\begin{array}{l}2.546 \mathrm{E}-9- \\
2.546 \mathrm{E}-9\end{array}$ \\
\hline Hydroureter & 128 & 0.251 & 0.667 & $0.334-1.331$ & $0.002^{*}$ & 0.329 & $0.161-0.675$ & 0.308 & 0.501 & $0.132-1.893$ \\
\hline Hydronephrosis & 255 & $0.034^{*}$ & 0.432 & $0.199-0.937$ & $0.000^{*}$ & 0.126 & $0.048-0.329$ & 0.667 & 0.719 & $0.160-3.227$ \\
\hline $\begin{array}{l}\text { In creased parenchymal } \\
\text { echogenicity }\end{array}$ & 36 & 0.918 & 0.934 & $0.254-3.463$ & $0.005^{*}$ & 0.188 & $0.058-0.608$ & 0.594 & 0.626 & $0.112-3.508$ \\
\hline Cortical thinning & 7 & 0.406 & 0.486 & $0.089-2.661$ & $0.012^{*}$ & 0.058 & $0.006-0.536$ & - & 4.085E-9 & $\begin{array}{l}4.085 E-9- \\
4.085 E-9\end{array}$ \\
\hline Swelling & 13 & 0.997 & 21629240.33 & $0.000^{-b}$ & 0.740 & 0.717 & $0.101-5.106$ & 0.550 & 0.427 & $0.026-6.935$ \\
\hline Bladder wall thickening & 9 & 0.810 & 1.312 & $0.143-12.045$ & 0.621 & 2.025 & $0.124-33.079$ & - & 2304517382 & $\begin{array}{r}2304517382- \\
2304517382\end{array}$ \\
\hline $\begin{array}{l}\text { No differentiation at } \\
\text { corticomedullary junciton }\end{array}$ & 2 & - & 23537545.99 & $\begin{array}{l}23537545.99- \\
23537545.99\end{array}$ & - & 447375924.7 & $\begin{array}{l}447375924.7- \\
447375924.7\end{array}$ & - & - & - \\
\hline Renal pelvic wall thickening & 1 & - & 21079462.02 & $\begin{array}{l}21079462.02- \\
21079462.02\end{array}$ & - & 13293918.64 & $\begin{array}{l}13293918.64- \\
13293918.64\end{array}$ & - & - & - \\
\hline CAKUT & 25 & $0.048^{*}$ & 0.354 & $0.127-0.989$ & 0.926 & 0.936 & $0.232-3.777$ & 0.998 & $1.867 E-9$ & $0.000-$ \\
\hline Normal finding & 527 & 0.879 & 0.934 & $0.386-2.258$ & 0.209 & 2.082 & $0.664-6.532$ & 0.557 & 2.082 & $0.664-6.532$ \\
\hline
\end{tabular}

If there was more than one RBUS finding in one patient, they were counted separately. aRS, acquired renal scarring; CAKUT, congenital anomaly of the kidney and urinary tract; HG VUR, high-grade vesicoureteral reflux; No., number of patients; RBUS, renal sonogram findings; rUTI, urinary tract infection recurrence; ${ }^{*}, P<0.05{ }^{b}$, systemically missing value; E, Exponent; ${ }^{C}$, systemically missing value

Table 3. Bivariate logistic regression analysis with odds ratio to predict urinary tract infection recurrence, high-grade vesicoureteral reflux, and acquired renal scarring according to various parameters compared with that obtained for abnormal renal sonogram findings.

\begin{tabular}{cccccccccc}
\hline & rUTI & OR & $95 \% C l$ & HGVUR & OR & $95 \% C l$ & aRS & OR & $95 \% C l$ \\
\hline Age & 0.294 & 1.01 & $0.995-1.018$ & 0.640 & 1.00 & $0.978-1.014$ & 0.847 & 1.00 & $0.978-1.018$ \\
Sex & 0.738 & 0.91 & $0.523-1.582$ & 0.789 & 1.12 & $0.491-2.550$ & 0.401 & 1.50 & $0.584-3.834$ \\
Abn RBUS & 0.228 & 1.39 & $0.816-2.354$ & $0.024^{*}$ & 2.64 & $1.139-6.124$ & $0.031^{*}$ & 2.58 & $1.089-6.091$ \\
Abn DMSA & 0.969 & 1.01 & $0.571-1.792$ & $0.003^{*}$ & 5.53 & $1.796-17.039$ & 0.094 & 0.37 & $0.114-1.186$ \\
\hline VUR & $0.045^{*}$ & 2.14 & $1.017-4.515$ & 0.990 & - & $0.000--$ & 0.383 & 0.61 & $0.202-1.846$ \\
HG VUR & $0.003^{*}$ & 3.47 & $1.533-7.870$ & - & - & - & $0.017^{*}$ & 4.64 & $1.319-16.337$ \\
rUTI & - & - & & $0.001^{*}$ & 4.27 & $1.772-10.281$ & $0.002^{*}$ & 4.82 & $1.758-13.198$ \\
\hline
\end{tabular}

Abn RBUS, abnormal renal sonogram findings; Abn DMSA, abnormal finding on 99m-technetium dimercaptosuccinic acid renal scan; aRS, acquired renal scarring; HG VUR, high-grade vesicoureteral reflux; OR, odds ratio; rUTI, urinary tract infection recurrence; * $P<0.05$

the first febrile UTI were not more likely to develop more frequent UTI than children with normal RBUS findings.

Gordon et al. reported that uroepithelial thickening is associated with increased risk of high grade VUR and is an abnormal finding warranting VCUG ${ }^{11)}$. However, in this study, renal pelvic wall thickening on RBUS was not associated with risk of high grade VUR. Kovanlikaya et al. reported that normal RBUS is rare with high grade VUR and that reflux grade was significantly associated with the degree of hydronephrosis ${ }^{12}$. Therefore, they suggested that RBUS is a good diagnostic screening tool for VUR ${ }^{12}$. However, Massanyi et al. reported that RBUS has poor sensitivity and negative predictive value for detecting high grade VUR in patients under 2 years who present with a febrile $\mathrm{UTI}^{13)}$. In this study, presence of a small kidney, hydroureter, hydronephrosis, cortical thinning, and increased parenchymal echogenicity were significant factors to predict high grade VUR. However, these factors' respective ORs were lower $(0.019,0.329,0.126,0.058$, and 0.188 , respectively) compared to cases with normal RBUS findings (OR 2.082). Therefore, children with hydroureter, hydronephrosis, cortical thinning, or increased parenchymal echogenicity at the first febrile UTI were not more likely to experience high grade VUR than children with 
normal RBUS findings.

There has been no previous literature on evaluation of the RBUS parameters associated with development of acquired renal scarring. This study showed that no RBUS findings were associated with acquired renal scarring. In order to confirm the presence of acquired renal scarring, a DMSA scan should be performed if clinically appropriate, regardless of the number of UTI recurrences or the presence of VUR. The authors suggest that a DMSA scan should be performed at least after the second episode of febrile UTI, except in cases with congenital renal scarring, regardless of RBUS findings at the first febrile UTI, because early detection of the presence of acquired renal scarring is a more sensitive indicator for surgical management of VUR than early detection of high grade VUR itself.

This study had some shortcomings. Data for this study was analyzed retrospectively. A sterile urine bag was used for urine culture in children under 2 years of age. RBUS was performed during the period of acute infection. Relatively few cases that received follow-up DMSA renal scan.

In summary, among RBUS findings, hydronephrosis and CAKUT predict UTI recurrence. Presence of a small kidney, hydroureter, hydronephrosis, cortical thinning, and increased parenchymal echogenicity predict highgrade VUR. However, compared to cases with normal RBUS finding, these factors are not useful parameters to predict UTI recurrence or high-grade VUR. RBUS is a necessary tool to exclude severe congenital renal scarring, obstructive uropathy, and renal abscess at the first incidence of febrile UTI. Furthermore, RBUS is clinically helpful in determining whether the next imaging study should be done.

\section{Conflict of Interest}

The authors declare that they have no conflict of interest.

\section{Financial Disclosure}

The authors declare that no financial or material support was taken for this research.

wastaken for this research.

\section{References}

1. Subcommittee on Urinary Tract Infection. Steering Committee on Quality Improvement and Management, Roberts KB. Urinary tract infection: clinical practice guideline for the diagnosis and management of the initial UTI in febrile infants and children 2 to 24 months. Pediatrics 2011;128:595-610.

2. Roberts JA. Experimental pyelonephritis in the monkey, part III: pathophysiology of ureteral malfunction induced by bacteria. Invest Urol 1975;13:117-20.

3. Fernbach SK, Maizels M, Conway JJ. Ultrasound grading of hydronephrosis: introduction to the system used by the Society for Fetal Urology. Pediatr Radiol 1993;23:478-80.

4. Han BK, Babcock DS. Sonographic measurements and appearance of normal kidneys in children. Am J Roentgenol 1985;145: 611-6.

5. Shaikh N, Ewing AL, Bhatnagar S, Hoberman A. Risk of renal scarring in children with a first urinary tract infection: a systemic review. Pediatrics 2010;126:1084-91.

6. Chen MJ, Cheng HL, Chiou YY. Risk factors for renal scarring and deterioration of renal function in primary vesico-ureteral reflux children: a long-term follow-up retrospective cohort study. PLoS One 2013;8:e57954. doi:10.1371/journal.pone.0057954. Epub 2013 Feb 28.

7. Panaretto K, Craig J, Knight J, Howman-Giles R, Sureshkumar P, Roy $L$. Risk factors for recurrent urinary tract infection in preschool children. J Paediatr Child Health 1999;35:454-9.

8. Soliman NA, Saif A, Hamid AA, Moustafa H. Renal duplex doppler ultrasonography in patients with recurrent urinary tract infection. Saudi J Kidney Dis Transpl 2009;20:816-21.

9. Katzir Z, Witzling M, Nikolov G, Gvirtz G, Arbel E, Kohelet D, et al. Neonates with extra-renal pelvis: the first 2 years. Pediatr Nephrol 2005;20:763-7.

10. Juliano TM, Stephany HA, Clayton DB, Thomas JC, Pope JC $4^{\text {th }}$, Adams MC, et al. Incidence of abnormal imaging and recurrent pyelonephritis after first febrile urinary tract infection in children 2 to 24 months old. J Urol 2013;190(4 supp):1505-10.

11. Gordon ZN, McLeod DJ, Becknell B, Bates DG, Alpert SA. Uroepithelial thickening on sonography improves detection of highgrade vesicoureteral reflux in children 2-24 months presenting with first febrile urinary tract infection. J Urol 2015 May 8. dio: 10.1016/j.juro.2015.05.001. [Epub ahead of print]

12. Kovanlikaya A, Kazam J, Dunning A, Poppas D, Johnson V, Medina $C$, et al. The role of ultrasonography in predicting vesicoureteral reflux. Urology 2014;84:1205-10.

13. Massanyi EZ, Preece J, Gupta A, Lin SM, Wang MH. Utility of screening ultrasound after first febrile UTI among patients with clinically significant vesicoureteral reflux. Urology 2013;82:905-9. 